\title{
Hilfestellung bei der Entwicklung einer Strategie für Digital Credentials im Bildungswesen
}

\section{Matthias Gottlieb und Hans Pongratz}

\section{Zusammenfassung}

Der Beitrag trägt zur Hilfestellung bei der Bewertung und Strategieentwicklung von Digital Credentials anhand einer Methodik mit definierten Zielen und der Analyse von bestehenden Fragestellungen bei. So erweitern die Ergebnisse des HFD-Workshops die Forschungsergebnisse Vogelsang et al. (2019), um die Anwendung in dem speziellen Szenario der Digital Credentials und zeigen, dass die Forschung der Wirtschaftsinformatik als Bindeglied eine Schlüsseldisziplin darstellt, die es den Nutzenden ermöglicht mit soziotechnischen Systemen einen Beitrag zu leisten. Wissenschaftler*innen beispielsweise aus den Gebieten der Akzeptanz- und Diffusionsforschung, der Psychologie können die Ergebnisse verwenden, um in einen Diskurs für weitere methodische Anwendungen in dem Bereich der Digital Credentials einzutreten. So können nutzerbasierte Systeme mit hoher Akzeptanz erforscht und für die Praxis geschaffen werden, um den dringenden Mehrwert durch die Digitalisierung besonders im Bereich der kleinen und mittelständischen Unternehmen zu erhalten. Auch die anwendungsorientierte Forschung im Bereich der Informatik kann Szenarien und technische Methoden erforschen und mittels Demonstratoren deren Machbarkeit aufzeigen.

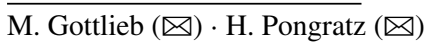

Technische Universität München (TUM), München, Deutschland

E-Mail: matthias.gottlieb@tum.de

H. Pongratz

E-Mail: pongratz@tum.de 


\section{Schlüsselwörter}

Bildungsnachweis • Digital Credentials • Digitalisierung •

Hochschulstrategie • Einflussfaktoren • Erfolgsfaktoren • Hilfestellung •

Strategie

\section{$1 \quad$ Einführung Digital Credentials}

Digital Credentials sind digitale Nachweise im Bildungswesen. Bildungseinrichtungen wie Hochschulen und Universitäten werden durch die Digitalisierung der Gesellschaft tief greifend verändert. Ausgelöst durch die Coronapandemie und die vielfältigen, länderspezifischen Ausgangsbeschränkungen hat sich die Notwendigkeit der Digitalisierung stark beschleunigt. Hinzu kommt die Art und Weise, wie akademische Zeugnisse und Abschlussdokumente ausgestellt, verteilt und verwaltet werden. Insellösungen einzelner Hochschulen sind trotz Bildungsföderalismus und Hochschulautonomie in Deutschland dabei aber nicht zielführend, da die nachhaltige elektronische Weiterverarbeitung von digitalen Bildungsnachweisen (Digital Credentials), die von den Studierenden gesammelt werden, bei zukünftigen Arbeitgebern, anderen Bildungseinrichtungen und staatlichen Stellen ohne eine standardisierte Lösung und ohne eine kritische Masse an Nutzer*innen nicht sichergestellt werden kann.

Dieser Beitrag soll explorativ eine Hilfestellung bei der Bewertung und Strategieentwicklung von Digital Credentials anhand einer Methodik mit definierten Zielen, der Analyse von bestehenden Fragestellungen mit ihren Stärken und Schwächen sowie abgeleiteten und diskutierten Erfolgsfaktoren für Hochschulen zur Vorbereitung, Bewertung und Bewältigung von Digital Credentials aufzeigen.

$\mathrm{Zu}$ den entscheidenden Herausforderungen der Entwicklung einer Hochschulstrategie für den Einsatz von Digital Credentials ist die Beachtung der Mehrdimensionalität von soziotechnischen Systemen (vgl. Krcmar 2015, ${ }^{1}$ S. 22; Lehner et al. 2008, S. 42; WKWI 2011), die durch die Digitalisierung in den Vordergrund rückt. Die Dimensionen (Bode et al. 2007) reichen dabei von der Nutzbarkeit des Systems, der Akzeptanz der Technologie, der Technologie als solcher selbst und der Erreichung einer kritischen Masse bis hin zu rechtlichen Fragestellungen des Datenschutzes und der Datensicherheit. Allein die Betrachtung der Technologie impliziert mehrere Möglichkeiten der Kombination unterschiedlich eingesetzter technischer Ressourcen und Methoden (Stock und Seliger 2016). Dies verdeutlicht die Komplexität der Thematik.

\footnotetext{
${ }^{1}$ Beispielsweise sind Informationssysteme soziotechnische Systeme.
} 
Ein weiterer Erfolgsfaktor für die digitale Transformation ist die Qualifikation der jeweiligen Beschäftigten (BMWi 2018). Beschäftigte und Organisationen müssen mit den neuen Rahmenbedingungen der Digitalisierung Schritt halten. Ideen von Beschäftigten können unter anderem genutzt werden, um einen Innovationsvorsprung sicherzustellen (Vogelsang et al. 2019). Die Digital Credential Initiative (MIT 2019) leistet nicht nur einen Beitrag zur Beschleunigung der Verwaltungsprozesse (beispielsweise bei Beglaubigungen) für den wichtigen Mittelstand in Deutschland, sondern unterstützt auch große Unternehmen und die Start-up-Szene. Die Rahmenbedingungen und die Initiative sind erste Bausteine zur Sicherung des Innovationsvorsprungs.

Die Digital Credential Initiative (MIT 2019), inzwischen das Digital Credentials Consortium (DCC) (MIT 2020), hat das Ziel, vertrauenswürdige Standards für die Erstellung, Darstellung und Verifizierung akademischer Bildungsnachweise zu entwickeln (HFD 2019; MIT 2019). Erste von Mitgliedern des DCC entworfene Prototypen sind in der Entwicklung und Erprobung. Zur Sicherstellung der Übertragbarkeit und Kompatibilität der Arbeiten wurde eine eigene öffentliche Education Task Force im Rahmen der World Wide Web Consortium (W3C) Credentials Community Group (CCG) Verifiable Credentials mit wöchentlichen Abstimmungsrunden eingerichtet (Duffy et al. 2020a).

\section{$2 \quad$ Aktueller Stand Digital Credentials}

In diesem Abschnitt geben wir einen knappen Überblick über Begrifflichkeiten und Entwicklungen in verschiedenen Kontexten von digitalen Bildungsnachweisen, die im internationalen Umfeld auch als Digital Credentials bezeichnet werden. Aufgrund der Komplexität des Themas können nur einzelne, plakative Beispiele und Entwicklungen genannt werden, ein Anspruch auf Vollständigkeit besteht nicht. Abb. 1 zeigt die Einordnung des Begriffs Digital Credentials in unterschiedliche Kontexte.

\subsection{Definition der Begrifflichkeiten}

Digital Credentials sind die digitalen Äquivalente zu Papierdokumenten und anderen materiellen Objekten, die von vertrauenswürdigen Parteien ausgegeben werden (Brands 2002).

Digital Credentials sind digitale Repräsentationen eines traditionell papierbasierten Zertifikats. Sie beweisen, dass ihre Inhaber*innen die spezifischen 


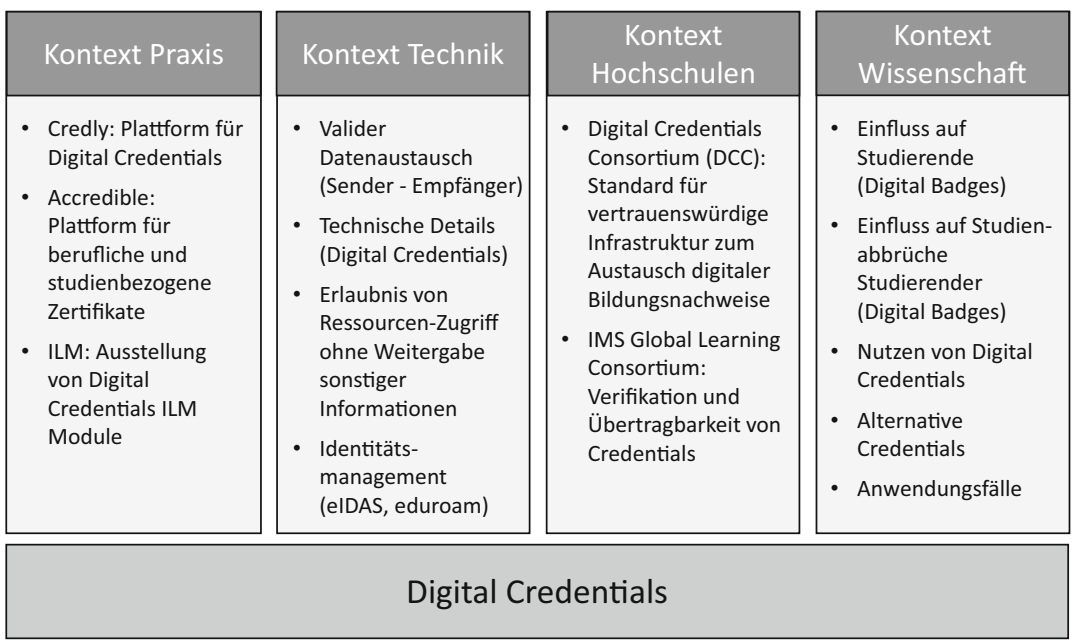

Abb. 1 Einordnung des Begriffs Digital Credentials in unterschiedliche Kontexte. (Eigene Darstellung)

Voraussetzungen zur Erreichung des jeweiligen Credentials erfüllt haben. Dabei stellen Digital Credentials eine zuverlässige Möglichkeit dar, Zertifikate in sozialen Netzwerken oder per E-Mail zu teilen. Arbeitgeber*innen können Zertifikate einfach und schnell verifizieren. Darüber hinaus erlauben ihnen Digital Credentials eine bessere Einschätzung des Zertifikats, da zusätzliche Informationen zur ausstellenden Organisation und $\mathrm{zu}$ den Voraussetzungen für den Erhalt bereitgestellt werden (Kleinhenz und Werding 2013; W3C Working Group 2019).

Digital Badges sind zur Vervollständigung der begrifflichen Abgrenzung zu nennen. Sie sind im Kontext Digital Credentials als ein Abzeichen oder eine Plakette für beispielsweise eine Konferenzteilnahme oder eine Begutachtung anzusehen. Sie können auch für die granulare Dokumentation des Erwerbs einer Kompetenz oder Lehrveranstaltungsteilnahme verwendet werden. Folglich ist ein Digital Credential von größerer Tragweite als ein Digital Badge (Accredible 2020; EDUCAUSE 2019). 


\subsection{Kontext Praxis}

Die Firma Credly stellt mit Credly eine Plattform für Digital Badges im Arbeitsumfeld zur Verfügung (Credly 2020). Dabei können Unternehmen eigene Digital Badges durch das Credential Dashboard erstellen oder sich bei der Einrichtung eines Leistungsanerkennungssystems von Fachleuten unterstützen lassen. Digital Badges können ausgegeben und von Inhabenden in sozialen Netzwerken geteilt werden. Weiterhin kann die Nutzung des Credentials-Programms anhand von Kennzahlen analysiert werden (Bange et al. 2013). Accredible stellt eine weitere Plattform dar, die berufliche und studienbezogene Zertifikate im Austauschformat Open Badges ausstellt (Accredible 2020; Gadatsch 2012).

Durch die schnelle Weiterentwicklung des Arbeitsumfelds ist eine kontinuierliche Fortbildung essenziell, um im Arbeitsmarkt wettbewerbsfähig zu bleiben. Als weitere Kategorie finden sich hier sogenannte Alternative Credentials, die der bedarfsgerechten Zertifizierung von Wissen und Kompetenzen im beruflichen Umfeld dienen (Observatory of Educational Innovation und Tecnológico de Monterrey 2019). $60 \%$ der Fachkundigen aus der Industrie glauben an einen Trend hin zu einer kompetenzbasierten anstelle einer abschlussbasierten Auswahl von zukünftigen Mitarbeitenden. $57 \%$ der Fachleute bestätigen, dass Arbeitgeber*innen mehr Wert auf alternative oder Microcredentials anstatt vergleichbarer Optionen legen. Universitäten sind sich dieses Trends bewusst und so führen erste Institutionen, wie beispielsweise die University of Texas, bereits Microcredentials ein (Digital Marketing Institute o. J.).

Folgende Aspekte scheinen bei der Einführung von Digital Credentials in Institutionen beachtet zu werden: die Entwicklung einer klaren Strategie, die den gesamten Lebenszyklus von Digital Credentials abdeckt, darüber hinaus die Erweiterung des eigenen Portfolios durch geeignete Fachinstitute und Partnerinstitute, um den Wert und die Integrität der eigenen Microcredentialangebote zu erhöhen. Auch die Ausstellung von Digital Credentials nach einheitlichen Standards, indem die Mitarbeitenden bezüglich dieser Standards geschult werden, wird berücksichtigt. Institute achten auf die Etablierung eines kontinuierlichen Verbesserungsprozesses für Digital Credentials, um hinsichtlich zukünftiger Trends auf dem aktuellen Stand zu bleiben, bei neuen Entwicklungen mithalten zu können und den Wert ihrer Digital Credentials nachhaltig sicherzustellen (Digital Marketing Institute o. J.). 


\subsection{Kontext Technik}

Es wurden bereits verschiedene Patente für Verfahren im Bereich der Digital Credentials angemeldet (Young 2019). Delegating Digital Credentials beruht auf einem Patent für ein System von Brickwell et al. (2008), das den Austausch von Daten zwischen Sender*in und Empfänger*in gewährleistet, indem das System die Validität der Daten überprüft und den Validitätsnachweis an die/den Empfänger*in übermittelt sowie der/dem Sender*in die Übermittlung des Validitätsnachweises bestätigt. Maher (2000) nennt ein weiteres Patent, um die folgenden elektronischen Transaktionen zu ermöglichen: Ausstellung eines Langzeitzertifikats (Credential), Validierung der etwas innehabenden Person dieses Langzeitzertifikats, Zusendung eines Kurzzeitzertifikats (Credential) an die innehabende Person. Des Weiteren können das Risiko der Inhabenden sowie weitere Informationen im Kurzzeitzertifikat gespeichert werden (Maher 2000).

Neben den Patenten stehen Methoden und technische Umsetzungen im Fokus. Barber und De Payne (2012) präsentieren eine Methode zur Bereitstellung von Digital Credentials zur Verifizierung von Nutzenden in Onlineforen und sozialen Netzwerken. Zusätzlich stehen die Beschreibung technischer Details im Fokus, wie beispielsweise kryptographische Sicherheitsmaßnahmen bei der Ausstellung von Digital Credentials, die Einrichtung einer limitierten Nutzung von Credentials bei Medikamentenrezepten oder Kinotickets oder auch die Gewährung des Zugriffs von Dritten auf das Credential (Brands 2002). Hierfür muss ein Protokoll entwickelt werden, um den Zugriff auf Ressourcen zu erlauben, ohne dabei andere Informationen an die Zugriffsberechtigten weiterzugeben (Frikken et al. 2006). Eine der entscheidenden technischen Herausforderungen stellt das Identity Management dar (Corradini et al. 2007). Es sind keine interoperablen, flexiblen und politikgesteuerten Lösungen bekannt, die das Identitätsmanagement vollständig integrieren (Corradini et al. 2007). Allerdings finden nationale und europäische Lösungen basierend auf dem neuen Personalausweis und der europäischen (electronic IDentification, Authentication and trust Services) eIDAS-Verordnung (Verordnung [EU] Nr. 910/2014 2014) erste Anwendung in Deutschland. Der Vollständigkeit halber ist in diesem Zusammenhang der weltweit sehr erfolgreiche Ansatz eduGAIN $^{2}$ als föderiertes Identity Management für den Dienst eduroam ${ }^{3}$ des europäischen Forschungsnetzwerks GÉANT zu erwähnen.

\footnotetext{
${ }^{2}$ Vgl. https://edugain.org/. Zugegriffen: 02.07.2020.

${ }^{3}$ Vgl. https://de.wikipedia.org/wiki/Eduroam oder https://www.eduroam.org/. Zugegriffen: 02.07.2020.
} 


\subsection{Kontext Hochschulen}

Digitale Zeugnisse sind für Hochschulen relativ neu und werden bisher wissenschaftlich wenig diskutiert (Durant und Trachy 2017). Erste Ansätze des Massachusetts Institute of Technology (MIT) gab es hier bereits 2015/2016 mit Blockcert (MIT 2016). 2018 gründete sich das heutige Digital Credentials Consortium (DCC) als internationaler, hochschulübergreifender und nicht herstellergetriebener Zusammenschluss, um einen weltweiten Standard für eine vertrauenswürdige Infrastruktur zum Austausch digitaler Bildungsnachweise zu entwickeln (Duffy et al. 2020b).

Eine weitere Kollaboration ist das IMS Global Learning Consortium, das mit Bildungsinstitutionen, $K-12$ districts, Arbeitgeber*innen und Technologieanbietern zusammenarbeitet, um das zukünftige Eco-System zu entwickeln (IMS Global Learning Consortium Inc. 2020). Das Konsortium umfasst folgende Projekte: Verifikation und Übertragbarkeit von Credentials am Beispiel von sogenannten Open Badges, transkriptähnliche Daten, die die spezifischen Leistungen einer Person an einer Institution dokumentieren und den Austausch von Kompetenzinformationen im Rahmen des Competencies and Academic Standards Exchange (IMS Global Learning Consortium Inc. 2020) ermöglichen.

Hochschul- und organisationsübergreifend hat sich seit 2012 das Groningen Declaration Network (GDN) mit dem Leitgedanken „We Make Digital Student Data Portability Happen“ (Groningen Declaration o. J.) formiert. Stand April 2019 wurde die Groningen-Erklärung von 98 Organisationen aus 27 verschiedenen Ländern und von zwei internationalen Netzwerken unterzeichnet. Im Rahmen des jährlichen Kongresses werden Vorhaben und Umsetzungen im Kontext des Studierenden-Datenaustausches vorgestellt, allerdings (zumindest bislang) keine gemeinsamen Standards erarbeitet.

\subsection{Kontext Wissenschaft}

Im Kontext Wissenschaft wird der potenzielle Nutzen von Digital Credentials untersucht, indem Studierende im Studium angesprochen werden, das Lernen durch Erfahrung gefördert und das Teilen von Zertifikaten vereinfacht wird. Studierende bewerteten Digital Credentials als Möglichkeit, sich von anderen Studierenden zu differenzieren und relevante Kompetenzen für den Arbeitsmarkt nachzuweisen. Außerdem empfanden Studierende den Prozess des Nachweises 
der erlangten Kompetenzen für den Erhalt der Digital Credentials als wertvoller, da ihnen damit eine bessere Selbstreflexion über die eigenen Kompetenzen möglich war (Miller et al. 2017).

Die Firma ILM arbeitet zusammen mit Credly an einer Lösung, um Digital Credentials für das erfolgreiche Absolvieren von ILM-Modulen anzubieten (ILM 2020). Diese Zertifikate können online geteilt werden. Digital Credentials ermöglichen auch hier die digitale Repräsentation von Kompetenzen oder Leistungen. Dabei enthalten sie Informationen, wie das Zertifikat erreicht wurde und wer das Zertifikat innehat (MIT 2019).

Einen Überblick der Literatur zu Learning Analytics ${ }^{4}$ und zur Vervollständigung und Abgrenzung von Digital Badges im Kontext der Minimierung von Studienabbrüchen im ersten Studienjahr gibt Mah (2016). Darin wird untersucht, wie Digital Badges die Fortsetzung des Studiums auf verschiedene Arten beeinflussen: Motivation, Anerkennung des Gelernten, Signalisierung von Erfolgen, Erfassung von Lernwegen. Auch der Einfluss von Digital Badges als Studienleistungsbewertungsmetrik anstatt der alleinigen Nutzung als Berechtigungsnachweis wird untersucht. Durch die Nutzung von Digital Badges zur Bewertung von Lernfortschritten können Studierende von Beginn eines Kurses an beim Lernen unterstützt werden, da kontinuierlich Feedback über den erreichten Fortschritt gegeben wird. Traditionell bekommen Studierende erst bei der Zwischen- oder Endklausur Rückmeldung darüber, wie viel Wissen sie sich bereits angeeignet haben. Weiterhin wurden positive Effekte auf die Einstellung gegenüber dem Kurs beobachtet, sobald Badges erreicht wurden. Jedoch konnte auch das gegenteilige Phänomen festgestellt werden, bei dem eine negative Einstellung entwickelt wurde, sobald nur wenige oder gar keine Badges erreicht wurden. Insgesamt ließ sich bei der Nutzung von Badges als Bewertungs-Tool jedoch eine höhere Motivation bei Studierenden beobachten (King et al. 2016).

\section{Methodisches Vorgehen}

Zur Beschreibung des methodischen Vorgehens wurde ein zweistufiger Ansatz gewählt, bei dem in einem ersten Schritt ein quantitativer Überblick gegeben und

\footnotetext{
${ }^{4}$ Nach Georg Siemens: ,[...] das Messen, Sammeln, Analysieren und Auswerten von Daten über Lernende und ihren Kontext mit dem Ziel, das Lernen und die Lernumgebung zu verstehen und zu optimieren“ (vgl. Jahn et al. 2019).
} 
im zweiten Schritt eine Vorgehensstrategie erarbeitet wird. Im Rahmen des zweiten Schrittes wurde in einem Workshop mit Fachleuten praxisbezogen und konkret gearbeitet.

Im ersten Schritt haben wir uns für die Suche nach relevanter Literatur am systematischen Vorgehen von Fettke (2006) orientiert. Wir fokussieren uns dabei auf die Breite, da aufgrund der relativ jungen Begrifflichkeit von „Digital Credentials“ in der Wirtschaftsinformatik (Duffy et al. 2020b; Gottlieb und Pongratz 2019) nur wenige Beiträge existieren. Die notwendige Tiefe wird über eine Vorwärts- und Rückwärtssuche erreicht. Die verwendeten Suchwörter sind: „digital credentials“ und ,digital credentials higher education“. Diese Kombination der Begrifflichkeiten ermöglicht die Fokussierung auf den Themenbereich der Digital Credentials im Umfeld des Bildungswesens. Nach Erfahrungen der Autoren ist der Begriff Digital Credentials stärker im englischsprachigen Raum zu finden, sodass wir uns daher darauf konzentriert haben.

Im zweiten Schritt haben wir einen Workshop im Rahmen der internationalen Strategie-Konferenz „Strategies Beyond Borders - Transforming Higher Education in a Digital Age" des Hochschulforums Digitalisierung (HFD) und des Deutschen Akademischen Austauschdiensts (DAAD) im Dezember 2019 durchgeführt. Am englischsprachigen Workshop nahmen über 30 Personen aus dem internationalen Hochschulleitungskreis wie auch aus Politik, Wirtschaft und Industrie teil. Die Teilnahme am Workshop erfolgte nach Interesse. Die teilnehmenden Personen waren intrinsisch motiviert und können als Fachkräfte betrachtet werden.

Um eine Strategie für Digital Credentials im Bildungswesen aufzuzeigen, wurde ein vierstufiger Ansatz gewählt. Die Entscheidung für diese Methode gründete sich auf zwei Erkenntnissen: Zum einen wurde erwartet, dass wegen der schnellen Veränderung in der Digitalisierung qualitative Methoden geeigneter als quantitative sind (Vogelsang et al. 2019), und zum anderen wurde von qualitativen Methoden erwartet, dass sich mit ihnen komplexe Interaktionen zwischen Technologien, Organisationen und beteiligten Personen erforschen lassen (Dubé und Paré 2003; Palvia et al. 2004; Vogelsang et al. 2019; Walsham 2006).

Die vier Stufen des Ansatzes gliedern sich wie folgt:

1. Als erste Stufe verwendeten wir die STEP-Analyse, um das Umfeld einer Organisation zu analysieren. Dieses Modell prüft Trends, die das Kerngeschäft einer Organisation nachhaltig prägen könnten. Die STEP-Analyse berücksichtigt folgende Dimensionen:

- Soziale Einflüsse

- Technische Einflüsse 
- Wirtschaftliche Einflüsse

- Politische Einflüsse

In seiner Gesamtheit betrachtet dieses Modell der Strategieentwicklung sowohl externe als auch interne Perspektiven und Ressourcen. Die Herausforderung lautet dabei, beide Seiten gleichgewichtig miteinzubeziehen. Diese Sichtweise wird auch als ganzheitliche Strategie bezeichnet.

2. Auf der zweiten Stufe leiten sich Erfolgsfaktoren aus den Einflüssen der ersten Stufe ab. Die Erfolgsfaktoren enthalten Messgrößen wie Wert, Methode, Frequenz und verantwortliche Person oder verantwortlicher Personenkreis. Zusätzlich werden die identifizierten Erfolgsfaktoren der Expert*innen mit den Ergebnissen aus der Literatursuche für eine validere Aussagekraft untermauert.

3. Auf der dritten Stufe kann auf Grundlage von Stufe zwei die Strategie entwickelt werden. Dazu werden geeignete Handlungsfelder identifiziert.

4. Auf der vierten Stufe wird die Strategie mit möglichen Anwendungsfällen überprüft. Das bedeutet, dass sowohl im einzelnen Handlungsfeld aus der dritten Stufe als auch im gesamten Kontext die Mehrdimensionalität validiert wird.

Um eine gegenseitige Beeinflussung der Teilnehmenden möglichst gering zu halten und trotzdem interaktiv zu arbeiten, verwendeten wir die Idee von World Cafés in der Hinsicht, dass für jede Dimension eine „Ecke“ zur Verfügung stand. Die Ergebnisse wurden mittels der Blitzlichtmethode präsentiert.

\section{Ergebnisse Workshop}

Im Workshop fanden aufgrund der begrenzten Zeitressourcen folgende zwei Stufen statt: (1) die STEP-Analyse der Einflüsse auf Digital Credentials und (2) die Erarbeitung von Erfolgsfaktoren basierend auf den Dimensionen der STEP-Analyse.

\subsection{STEP-Analyse: Einflussfaktoren auf Digital Credentials}

Die sozialen Einflussfaktoren umfassen nach Einschätzung der Fachleute heterogene Bildungswege. Das bedeutet, moderne Biografien sind nicht mehr linear, sondern bestehen aus einzelnen, individuellen Modulen des Lernens. Darüber hinaus sind verschiedene Institutionen an der Wissensvermittlung beteiligt. Der modulare Studienaufbau führt zu einer stärkeren Modularisierung der Bildung, 
die sich in formelles und informelles Lernen einteilen lässt. Die Sichtbarkeit der Module kann nach Einschätzung der Expert*innen durch Digital Credentials erhöht werden. Im sozialen Umfeld wird demnach ein digitaler Lebensstil entstehen. Dies führt zu Fragen nach Anerkennung und Akzeptanz von bestimmten Zertifikaten. Schließlich können diese von jeder Institution generiert werden. Der Vollständigkeit halber ist anzumerken, dass aktuelle Microcredentialprogramme wesentlich kostengünstiger als die Einschreibung in ein vollständiges Universitätsprogramm sind. Der qualitativ hochwertige Bildungsauftrag kann allerdings nur von den Hochschulen und den Universitäten übernommen werden.

Die technischen Einflussfaktoren sind laut den Fachleuten in der Persistenz von Institutionen und Technologie zu sehen. Dabei ist die Technologiemigration von zentraler Bedeutung. Bei der Authentifizierung sind sich die teilnehmenden Personen einig und befürworten mit Nachdruck kryptographische Ansätze. Als technische Lösung kommen sowohl zentralisierte als auch dezentralisierte Lösungen in Betracht, wobei ein Trend zur Dezentralisierung gesehen wird, während ein zentralisiertes Register bei einer europäischen oder gar weltweiten Variante als erforderlich angesehen wird. Zwecks Vertrauensbildung muss das System zuverlässig sein. Gerade die automatische Erkennung und der automatisierte Austausch von Digital Credentials werden von den Expert*innen als Novum eingeschätzt, das die Möglichkeit des Vertrauens des Menschen in die Maschine noch unter Beweis zu stellen hat. Unter der Annahme, dass die Sicherheit der Kommunikation in den sozialen Medien das Vertrauen in digitale Zertifikate fördert, stellte sich folgende weiterführende Forschungsfrage: Welchen Einfluss hat Social Media auf die Vertrauenswürdigkeit von digitalen Zertifikaten?

Die wirtschaftlichen Einflussfaktoren benannten die Fachleute mit der allgemeinen Fragestellung nach einer Struktur des Marktes für Digital Credentials. Diese Marktstruktur muss geschaffen werden, um den wirtschaftlichen Wert von Digital Credentials nachhaltig zu etablieren. Zusätzlich stellte sich die Frage nach dem Vorhandensein eines Marktes für die Prüfung von Zertifikaten. Insbesondere gab es Bedenken, ob Studierende bereit wären, für einen solchen Service zu zahlen, und wenn ja, unter welchen Bedingungen. Zusätzlich stellte sich die Frage, wer für die Infrastrukturkosten aufkommt und wie die Hintergründe für diese Entscheidung aussehen könnten.

Politische Einflussfaktoren sind beispielsweise die Pilotierung und Einführung des New Europass ${ }^{5}$ ab Juli 2020, die von den Expert*innen als eine wesentliche Einflussgröße gesehen werden. Dies hat mehrere Gründe: zum einen die

${ }^{5}$ Siehe: https://europass.cedefop.europa.eu/de/new-europass. Zugegriffen: 02.07.2020. 
politische Unterstützung über die gesamte EU hinweg und zum anderen den Willen der Mitgliedsstaaten, dies auch monetär zu unterstützen. Beim New Europass handelt es sich um eine europäische Infrastruktur für Digital Credentials (Europäische Kommision 2020). Mithilfe der für Institutionen und EU-Bürger*innen kostenlose Softwareplattform können digitale, fälschungssichere Qualifikationen und andere Lernnachweise ausgestellt, verwaltet und $\mathrm{zu}$ einem persönlichen Lebenslauf zusammengefasst werden (Europäische Kommision 2020).

Demgegenüber sehen die Expert*innen das European Credit Transfer System (ECTS) nur im Europäischen Hochschulraum. Es stellt sich so insbesondere die Frage nach dem Vertrauen in dieses System, wenn es einer globalen Akzeptanz bedarf. Zusätzlich erkennen die Expert*innen eine Herausforderung hinsichtlich des Datenschutzes über die Ländergrenzen hinweg. Insbesondere beim Vergleich zwischen der Europäischen Union (27 Mitgliedsstaaten), dem Europäischen Hochschulraum (48 Länder), den USA und teilweise föderalen Verschärfungen innerhalb Deutschlands zeigen sich enorme Unterschiede im Datenschutz.

\subsection{Erfolgsfaktoren für Digital Credentials}

Als soziale Erfolgsfaktoren nannten die Fachleute die sogenannte Upward Mobility, die als Bewegung von einem niedrigeren zu einem höheren sozialen und wirtschaftlichen Status verstanden wird. Die Wertschätzung und Anerkennung der Digital Credentials durch den Arbeitsmarkt sind für eine Durchdringung notwendig. Hinzu kommen die Frequenz und Anzahl der berechtigten und benötigten Beglaubigungsschreiben der einzelnen Personen. Hiernach richtet sich auch die Akzeptanz der Berechtigungsnachweise, die das Profil einer Person dann ausmachen. Für die Akzeptanz ist eine einfache Übertragung von Berechtigungsnachweisen und deren Manipulationssicherheit ein signifikanter Faktor. Die Beurteilung verantworten zum einen die beteiligten Personen, die hieraus mittels einer Portfolioverwaltung Nutzen ziehen, zum anderen tun dies die Emittent*innen im Sinne der Transparenz. Hingegen sehen die Fachleute die formelle und informelle Anerkennung von Fähigkeiten als Verhandlungssache an. Corradini et al. (2007) erörtern ausführlich das Identitätsmanagement, das im Zusammenhang mit Digital Credentials notwendig ist und einen entscheidenden Erfolgsfaktor darstellt.

Als technologische Erfolgsfaktoren erarbeiteten die Teilnehmer*innen des Workshops folgende Kriterien: Akzeptanz und Vertrauen; Normen zu Technologie, Verfahren und Inhalten; Transparenz im Sinne des Niveaus technologischer Sicherheit; Schnittstellen im Sinne der Belastbarkeit sowie eine Fallback-Option 
zur Vorbeugung von Ausfällen und Fragmentierung (Option zur Präsentation ausgewählter Abschlüsse) im Gegensatz zu lebenslangen Lernwegen (Lifetime Learning Pathways) (Reputation, Arbeitsbelastung, Dauer). Als weitere Erfolgsfaktoren im Bereich der Technologie innerhalb der digitalen Transformation benennen Vogelsang et al. (2019): Infrastruktur, Verlässlichkeit, Relevanz, Anpassungsfähigkeit, Vollständigkeit, Erreichbarkeit und Echtzeitdaten. Die Teilnehmer*innen bestätigten die Erkenntnisse von Vogelsang et al. (2019), die ebenfalls das Thema Sicherheit im Sinne von dem englischen Security anstelle von Safety behandeln. Corradini et al. (2007) stellen ein ganzheitliches Framework als notwendig dar, um Sicherheits-, Vertrauens- und Datenschutzaspekte auf verschiedenen Abstraktionsebenen und damit Richtlinien auf politischer Ebene zu schaffen, Probleme schnell zu identifizieren (zum Beispiel Missbrauch von Identitätsinformationen, Angriffe und Richtlinienverletzungen) und entsprechend zu reagieren.

Als Messgröße für den Durchdringungsgrad ist im Workshop von den Expert*innen die Anzahl der Personen, die ein solches digitales Zertifikat anerkennen, identifiziert worden. Zusätzlich kann die Messung der Akzeptanz beispielsweise mit den Theorien/Methoden zur Technologieakzeptanz (Davis 1986; Venkatesh und Bala 2008; Venkatesh und Davis 2000; Venkatesh et al. 2003) erfolgen.

Als wirtschaftliche Erfolgsfaktoren sehen die Fachleute den gesellschaftlichen Wert, der gerade in Deutschland durch die Steuerzahler*innen gegeben ist. So ist eine digitale Infrastruktur in der Beschaffung günstiger als eine analoge Infrastruktur. Infrastrukturkosten können also als ein Erfolgsindikator abgeleitet werden. Diese ergeben sich auch durch die schnellere Verfügbarkeit und die entfallenden Grenzkosten (Scheer 2016). Ein weiterer Wert entsteht für potenzielle Arbeitgeber*innen, die für den Service zahlen. So kann der Abgleich der Qualifikationen mit den Anforderungen der Arbeitgeber*innen an die Beschäftigungsfähigkeit als Indikator gesehen werden. Unabhängig davon wird ein Wert in KI-basierten Analysen von Berechtigungsnachweis-Datenbanken gesehen.

Als politische Erfolgsfaktoren werteten die Expert*innen die messbare politische Akzeptanz. Die politischen Rahmenbedingungen durch Akzeptanz nennen auch Corradini et al. (2007) als entscheidenden Faktor. Als mögliche Messgrößen identifizierten die Expert*innen die Anzahl an Ländern, die konkrete Gesetze beschließen, die Anzahl an Finanzierungsprogrammen für Digital Credentials, die Anzahl der Prototypen sowie die Behandlung der Thematik auf international anerkannten Konferenzen. 


\section{Diskurs, Zusammenfassung und Ausblick}

Im Gegensatz zur verwendeten STEP-Analyse betrachten Vogelsang et al. (2019) die Ergebnisse anhand von Erfolgsfaktoren basierend auf dem Modell von DeLone und McLean (1992). DeLone und McLean (1992) unterteilen die Erfolgsfaktoren in die drei Kategorien Organisation, Umgebung und Technologie. Die Umgebung spielt in der STEP-Analyse keine Rolle. Sie stellt jedoch ein entscheidendes Handlungsfeld im dritten Schritt der angewendeten Methode dar. Vogelsang et al. (2019) identifizieren Konnektivität, Transparenz, Kollaboration, Standards und hybride Wertschöpfung als Erfolgsfaktoren der Umgebung. Diese Erfolgsfaktoren, so hat der Workshop gezeigt, fallen im speziellen Anwendungsfall den Fachleuten zufolge unter den Bereich der Technologie. Dieser Beitrag erweitert die bisherigen Forschungserkenntnisse (DeLone und McLean 1992; Vogelsang et al. 2019) um die Anwendung im speziellen Szenario der Digital Credentials.

Die Ergebnisse des Workshops mit Fachleuten bestätigen die Erkenntnisse von Corradini et al. (2007) hinsichtlich Akzeptanz und Vertrauen. Aufgrund der Mehrdimensionalität des Themas sind die interne und externe Validität unter Berücksichtigung wissenschaftlicher Methoden sicherzustellen. Zusätzlich erweitern die Ergebnisse damit die Forschungserkenntnisse von Vogelsang et al. (2019) um die Anwendung im speziellen Szenario der Digital Credentials und zeigen, dass die Forschung der Wirtschaftsinformatik als Bindeglied eine Schlüsseldisziplin darstellt, die es den Nutzer*innen ermöglicht, mit soziotechnischen Systemen einen Beitrag zu leisten.

Wissenschaftler*innen beispielsweise aus den Gebieten der Akzeptanz- und Diffusionsforschung ${ }^{6}$ der Psychologie können die Ergebnisse verwenden, um in einen Diskurs über weitere methodische Anwendungen in dem Bereich der Digital Credentials einzutreten. So können nutzer*innenbasierte Systeme mit hoher Akzeptanz erforscht und für die Praxis geschaffen werden, um den dringend benötigten Mehrwert durch die Digitalisierung besonders im Bereich der kleinen und mittelständischen Unternehmen zu erhalten. Auch die anwendungsorientierte Forschung im Bereich der Informatik kann Szenarien und technische Methoden erforschen und mittels Demonstratoren deren Machbarkeit aufzeigen.

\footnotetext{
${ }^{6}$ Vgl. Davis (1986), Venkatesh und Davis (2000), Venkatesh und Bala (2008).
} 


\section{Literatur}

Accredible. (2020). Digital credentials. https://www.accredible.com/credentials/. Zugegriffen: 2. Juli 2020.

Bange, C., Grosser, T., \& Janoschek, N. (2013). Big Data Survey Europe: Nutzung, Technologie und Budgets europäischer Best Practice Unternehmen. Würzburg: BARC-Institut.

Barber, T. P., \& De Payne, L. (2012). USA Patent Nr. US 8,296,245 B2. USPTO: USPTO.

BMWi. (2018). Monitoring-Report Wirtschaft DIGITAL 2018. https://www.bmwi.de/Red aktion/DE/Publikationen/Digitale-Welt/monitoring-report-wirtschaft-digital-2018-lan gfassung.pdf?_blob=publicationFile\&v=4. Zugegriffen: 2. Juli 2020.

Bode, A., Borgeest, R., \& Pongratz, H. (2007). The ICT Strategy of the Technische Universität München. In Paper Presented at the Proceedings of 13th International Conference on European University Information Systems. Grenoble.

Brands, S. (2002). A technical overview of digital credentials. https://citeseerx.ist.psu.edu/ viewdoc/download?doi=10.1.1.16.3617\&rep=rep1\&type=pdf. Zugegriffen: 2. Juli 2020.

Brickwell, E. F., Deklotz, W., Glover, J. U., Premi, M. R., Wood, M. D., \& Shimoda, M. H. (2008). Delegating digital credentials. USPTO: Intel Corporation.

Corradini, F., Paganelli, E., \& Polzonetti, A. (2007). The e-Government digital credentials. International Journal Electronic Governance, 1(1), 17-37.

Credly. (2020). The common language of verified skills. https://info.credly.com/. Zugegriffen: 2. Juli 2020.

Davis, F. D. (1986). A technology acceptance model for empirically testing new end-user information systems - Theory and results. (Dissertation). Cambridge: MIT.

DeLone, W. H., \& McLean, E. R. (1992). Information systems success: The quest for the dependent variable. Information Systems Research, 3(1), 60-95.

Digital Marketing Institute. (o. J.). What do digital credentials mean for education? https://digitalmarketinginstitute.com/blog/what-do-digital-credentials-mean-foreducation. Zugegriffen: 2. Juli 2020.

Dubé, L., \& Paré, G. (2003). Rigor in information systems positivist case research: Current practices, trends, and recommendations. MIS Quarterly, 27(4), 597-636. https://doi.org/ $10.2307 / 30036550$.

Duffy, K. H., Andrieu, J., \& Allen, C. (2020a). CCG verifiable credentials for education task force telecon. https://w3c-ccg.github.io/meetings/2020-04-27-vc-education/. Zugegriffen: 2. Juli 2020.

Duffy, K. H., Pongratz, H., Schmidt, J. P., Chartrand, J., Freeman, S., Gallersdörfer, U., Lisle, M., Mühle, A., van Engelenburg, S., Rajagopal, K., Asthagiri, N., Bertazzo, M., Burke, M., Canavan, B., Cherubini, P., Chuang, I., Crittenden, C., DeFreece, D., DePauw, J., Dieffenbach, J., Epema, D. H. J., Escamilla, J., Gottlieb, M., Harmon, S., Heyer, O., Hidrogo, I., Kan, M., Kos, T., Leitner, H., van Leersum, N., Matkin, G., Moldoveneau, M., Pool, M., Puri, I. K., Renz, J., Saldivar, B., Sarma, S., Subirana, B., Sussenbach, M., Tan, T., Tingley, D., van Valkenburg, W., Wu, D., White, M., Wolf, A., \& Wong, W. (2020b). Building the digital credential infrastructure for the future. https://digitalcredentials.mit.edu/wp-content/uploads/2020/02/whitepaper-building-digital-credential-infrastructure-future.pdf. Zugegriffen: 2. Juli 2020. 
Durant, E., \& Trachy, A. (2017). Digital diploma debuts at MIT. https://news.mit.edu/2017/ mit-debuts-secure-digital-diploma-using-bitcoin-blockchain-technology-1017. Zugegriffen: 2. Juli 2020.

EDUCAUSE. (2019). Badges and Credentialing. https://library.educause.edu/topics/tea ching-and-learning/badges-and-credentialing. Zugegriffen: 2. Juli 2020.

Europäische Kommission. (2020). New Europass. https://ec.europa.eu/futurium/en/europass/ new-europass. Zugegriffen: 2. Juli 2020.

Fettke, P. (2006). State-Of-The-Art des State-Of-The-Art: Eine Untersuchung der Forschungsmethode „Review“ innerhalb der Wirtschaftsinformatik. Wirtschaftsinformatik, 48(4), 257-266.

Frikken, K., Atallah, M., \& Li, J. (2006). Attribute-based access control with hidden policies and hidden credentials. IEEE Transactions on Computers, 55(10), 1259-1270.

Gadatsch, A. (2012). Big data. wisu das Wirtschaftsstudium, 41(12), 1615-1621.

Gottlieb, M., \& Pongratz, H. (2019). Towards digital business process models in higher education institutions: A case study based on the onboarding of student employees. In Paper Presented at the 13th Mediterranean Conference on Information Systems. Neapel.

Groningen Declaration (o. J.). https://www.groningendeclaration.org/. Zugegriffen: 16.10.2020.

Hochschulforum Digitalisierung. (2019). Digital Credentials Initiative - neun Universitäten arbeiten zusammen an globaler Infrastruktur für Digital Credentials. https://hochschulfor umdigitalisierung.de/de/news/global-infrastructure-digital-academic-credentials. Zugegriffen: 2. Juli 2020.

ILM. (2020). Access your Credentials. https://www.i-1-m.com/learning-and-development/dig ital-credentials. Zugegriffen: 30. Apr. 2020.

IMS Global Learning Consortium Inc. (2020). Understanding digital credentials - Building value from an ecosystem of open standards. https://www.imsglobal.org/understandingdigital-credentials. Zugegriffen: 2. Juli 2020.

Jahn, S., Kaste, S., März, A., \& Stühmeier, R. (2019). Denkimpuls Digitale Bildung: Einsatz von Künstlicher Intelligenz im Schulunterricht. Berlin: Initiative D21 e.V. https://initiativ ed21.de/app/uploads/2019/05/d21-denkimpuls_schule_ki.pdf. Zugegriffen: 16.10.2020.

King, E., Steinkuehler, C., Gee, E. R., Herro, D., Holmes, J. B., Dickmann, E., \& Abramovich, S. (2016). Understanding digital badges in higher education through assessment. On the Horizon, 24(1), 126-131. https://doi.org/10.1108/oth-08-2015-0044.

Kleinhenz, G., \& Werding, M. (2013). Gesundheitswesen. https://wirtschaftslexikon.gabler. de/Archiv/55801/gesundheitswesen-v8.html. Zugegriffen: 2. Juli 2020.

Krcmar, H. (2015). Informationsmanagement (6., überarbeitete Aufl.). Berlin: Springer Gabler.

Lehner, F., Wildner, S., \& Scholz, M. (2008). Wirtschaftsinformatik: Eine Einführung (2. Aufl.). München: Hanser.

Mah, D.-K. (2016). Learning analytics and digital badges: Potential impact on student retention in higher education. Technology, Knowledge and Learning, 21(3), 285-305. https:// doi.org/10.1007/s10758-016-9286-8.

Maher, D. P. (2000). Method and apparatus using digital credentials and other electronic certificates for electronic transactions. Corp: A. T.

Miller, K. K., St. Jorre, T. J. d., West, J. M., \& Johnson, E. D. (2017). The potential of digital credentials to engage students with capabilities of importance to scholars and citizens. 
Active Learning in Higher Education, 21(1), 11-22. https://doi.org/10.1177/146978741 7742021.

MIT. (2016). Digital certificates project. https://certificates.media.mit.edu/. Zugegriffen: 2. Juli 2020.

MIT. (2019). Digital credentials. https://digitalcredentials.mit.edu/. Zugegriffen: 2. Juli 2020.

MIT. (2020). University-led digital credentials consortium explores technology for digital academic credentials. https://openlearning.mit.edu/news/university-led-digital-creden tials-consortium-explores-technology-digital-academic-credentials. Zugegriffen: 16. Okt. 2020.

Observatory of Educational Innovation, \& Tecnológico de Monterrey. (2019). Alternative credentials. eduTrends, 5. https://observatory.tec.mx/edu-trends-alternative-credentials. Zugegriffen: 2. Juli 2020.

Palvia, P., Leary, D., Mao, E., Midha, V., Pinjani, P., \& Salam, A. (2004). Research methodologies in MIS: An update. Communications of the Association for Information Systems, 14(1), 24.

Scheer, A.-W. (2016). Thesen zur Digitalisierung. In F. Abolhassan (Hrsg.), Was treibt die Digitalisierung? (S. 49-61). Wiesbaden: Gabler.

Stock, T., \& Seliger, G. (2016). Opportunities of sustainable manufacturing in industry 4.0. Procedia Cirp, 40, 536-541. https://doi.org/10.1016/j.procir.2016.01.129.

Venkatesh, V., \& Bala, H. (2008). Technology acceptance model 3 and a research agenda on interventions. Decision Sciences, 39(2), 273-315.

Venkatesh, V., \& Davis, F. D. (2000). A theoretical extension of the technology acceptance model: Four longitudinal field studies. Management Science, 46(2), 186-204.

Venkatesh, V., Morris, M. G., Davis, G. B., \& Davis, F. D. (2003). User acceptance of information technology: Toward a unified view. MIS Quarterly, 27(3), 425-478. https://www.jstor.org/discover/10.2307/30036540?uid=3737864\&uid=2\&uid= $4 \&$ sid $=21104510074511$. Zugegriffen: 2 . Juli 2020.

Verordnung (EU) Nr. 910/2014. (2014). Verordnung (EU) Nr. 910/2014 des Europäischen Parlaments und des Rates vom 23. Juli 2014 über elektronische Identifizierung und Vertrauensdienste für elektronische Transaktionen im Binnenmarkt und zur Aufhebung der Richtlinie 1999/93/EG. Official Journal of the European Union, L257/73. https://eur-lex. europa.eu/legal-content/DE/TXT/PDF/?uri=CELEX:32014R0910. Zugegriffen: 2. Juli 2020.

Vogelsang, K., Liere-Netheler, K., Packmohr, S., \& Hoppe, U. (2019). Success factors for fostering a digital transformation in manufacturing companies. Journal of Enterprise Transformation, 1-22. https://doi.org/10.1080/19488289.2019.1578839.

Walsham, G. (2006). Doing interpretive research. European Journal of Information Systems, 15(3), 320-330.

WKWI. (2011). Profil der Wirtschaftsinformatik, Ausführungen der Wissenschaftlichen Kommission der Wirtschaftsinformatik. In K. Kurbel, J. Becker, N. Gronau, E. Sinz, \& L. Suhl (Hrsg.), Enzyklopädie der Wirtschaftsinformatik - Online-Lexikon (4. Aufl.). München: Oldenbourg. https://www.enzyklopaedie-der-wirtschaftsinformatik.de/wi-enz yklopaedie/lexikon/uebergreifendes/Disziplinen\%20der\%20WI/Wirtschaftsinformatik/ profil-der-wirtschaftsinformatik/index.html/. Zugegriffen: 2. Juli 2020.

W3C Working Group. (2019). Verifiable credentials use cases. https://www.w3.org/TR/vcuse-cases/\#user-needs. Zugegriffen: 2. Juli 2020. 
Young, J. R. (2019). Who owns digital badges? A company's patent on credential system raises questions. EdSurge. https://www.edsurge.com/news/2019-03-12-who-owns-digitalbadges-a-company-s-patent-on-credentials-raises-questions. Zugegriffen: 2. Juli 2020.

Open Access Dieses Kapitel wird unter der Creative Commons Namensnennung 4.0 International Lizenz (http://creativecommons.org/licenses/by/4.0/deed.de) veröffentlicht, welche die Nutzung, Vervielfältigung, Bearbeitung, Verbreitung und Wiedergabe in jeglichem Medium und Format erlaubt, sofern Sie den/die ursprünglichen Autor(en) und die Quelle ordnungsgemäß nennen, einen Link zur Creative Commons Lizenz beifügen und angeben, ob Änderungen vorgenommen wurden.

Die in diesem Kapitel enthaltenen Bilder und sonstiges Drittmaterial unterliegen ebenfalls der genannten Creative Commons Lizenz, sofern sich aus der Abbildungslegende nichts anderes ergibt. Sofern das betreffende Material nicht unter der genannten Creative Commons Lizenz steht und die betreffende Handlung nicht nach gesetzlichen Vorschriften erlaubt ist, ist für die oben aufgeführten Weiterverwendungen des Materials die Einwilligung des jeweiligen Rechteinhabers einzuholen.

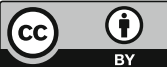

